REVIEW PAPER

\title{
Systematic review of systematic reviews for the management of urinary incontinence and promotion of continence using conservative behavioural approaches in older people in care homes
}

\author{
Brenda Roe, Lisa Flanagan \& Michelle Maden
}

Accepted for publication 18 November 2014

Correspondence to B. Roe:

e-mail: brenda.roe@edgehill.ac.uk

Brenda Roe PhD RN RHV

Professor of Health Research/ Honorary

Fellow

Evidence-based Practice Research Centre, Faculty of Health \& Social Care, Edge Hill University, Ormskirk, UK and Personal Social Services Research Unit, University of Manchester, UK

Lisa Flanagan BSc MBBch MRCP Consultant Community Geriatrician Countess of Chester Hospital NHS Foundation Trust, UK

Michelle Maden BA MA FHEA Clinical Information Specialist/Learning Services and Associate Tutor Faculty of Health \& Social Care, Edge Hill University, Ormksirk, UK
ROE B., FLANAGAN L. \& MADEN M. (2015) Systematic review of systematic reviews for the management of urinary incontinence and promotion of continence using conservative behavioural approaches in older people in care homes. Journal of Advanced Nursing 00(0), 000-000. doi: 10.1111/jan.12613

\footnotetext{
Abstract

Aim. To synthesize evidence from systematic reviews on the management of urinary incontinence and promotion of continence using conservative/behavioural approaches in older people in care homes to inform clinical practice, guidelines and research.

Background. Incontinence is highly prevalent in older people in care home populations.

Design. Systematic review of systematic reviews with narrative synthesis.

Data sources. Electronic searches of published systematic reviews in English using MEDLINE and CINAHL with no date restrictions up to September 2013. Searches supplemented by hand searching and electronic searching of Cochrane Library and PROSPERO.

Review methods. PRISMA statement was followed, as were established methods for systematic review of systematic reviews.

Results. Five systematic reviews of high quality were included, three specific to intervention studies and two reviewed descriptive studies. Urinary incontinence was the primary outcome in three reviews with factors associated with the management of urinary incontinence the primary outcome for the other reviews.

Conclusion. Toileting programmes, in particular prompted voiding, with use of incontinence pads are the main conservative behavioural approach for the management of incontinence and promotion of continence in this population with evidence of effectiveness in the short term. Evidence from associated factors; exercise, mobility, comorbidities, hydration, skin care, staff perspectives, policies and older people's experiences and preference are limited. The majority of evidence of effectiveness are from studies from one country which may or may not be transferable to other care home populations. Future international studies are warranted of complex combined interventions using mixed methods to provide evidence of effectiveness, context of implementation and economic evaluation.
} 
Keywords: associated factors with incontinence, care homes, conservative behavioural approaches, evidence synthesis, management of incontinence, nursing homes, older people, promotion of continence, systematic reviews, toileting programmes, urinary incontinence

\section{Why is the research or review needed?}

- Urinary incontinence is highly prevalent in older people in long-term care; including nursing, residential or care homes and aged care facilities in the community.

- The costs of managing incontinence in terms of staff time, resources, aids and appliances are high yet economic evaluations remain few.

- There is limited but emerging evidence of effectiveness from systematic reviews of conservative/behavioural approaches for the management of urinary incontinence which form the main focus of nursing care.

\section{What are the key findings?}

- Evidence synthesis confirms that empirical research of toileting programmes, in particular prompted voiding, comprise the main interventions and approaches used for managing incontinence and promoting continence in older people in care homes. There is some evidence of effectiveness in the short term.

- More interventions studies, predominantly trials, are available than descriptive observational studies. Irrespective of their design, studies are heterogeneous but indicate toileting and use of incontinence aids predominate with more recent studies being of higher methodological quality.

- Few studies are available on economic evaluations (stand alone or in addition to or follow on from effectiveness trials) and studies of maintaining continence in older people in care homes lacking.

How should the findings be used to influence policy/ practice/research/education?

- This evidence synthesis is the first of its kind and is useful to inform current national and international guidelines and consensus consultations.

- Future studies that combine complex interventions using standardized outcomes and mixed methods with qualitative studies embedded including both implementation and economic evaluations are warranted. Studies should adhere to established international methodological and publication standards.

- Nursing practice and values should reaffirm a focus on 'embodied' care, i.e. meeting the essential basic needs of older people in terms of mobility, elimination, nutrition, hydration and hygiene while preserving dignity. Involving older people as partners in compassionate care is paramount.

\section{Introduction}

Urinary incontinence (UI) is a prevalent condition in older people in care home populations in many countries with estimates ranging from 31-70\% (Ouslander \& Schnelle 1995, Sgadari et al. 1997, McGrother et al. 2003, DuBeau et al. 2009). Incontinence is defined as 'the involuntary or inappropriate passing of urine and/or faeces that has an impact on social functioning or hygiene. It also includes nocturnal enuresis (bed wetting) (DH 2000, p7). UI is associated with pressure ulcers (Spector 1994, Berlowitz et al. 1997), urinary tract infection (UTI)(Richardson \& Hriez 1995), falls (Kron et al. 2003, Foley et al. 2012) and diminished quality of life (DuBeau et al. 2006). UI is also prevalent in people with faecal incontinence (FI) (Diokno et al. 1986, Aggazzotti et al. 2000), stroke (Jorgensen et al. 2005, Dumoulin et al. 2007, Williams et al. 2012), dementia (Grant et al. 2013), heart failure (Palmer 2009) and diabetes (Brown et al. 2006). Managing UI in care homes incurs both personal and institutional costs related to staff time, aids and appliances and laundry costs ( $\mathrm{Hu}$ et al. 1989, Schnelle et al. 1989, Hu et al. 1990, McGrother et al. 2003, DuBeau et al. 2009). In the US costs of caring for residents with UI is estimated at $\$ 10,000$ per patient per year (Borrie \& Davidson 1992, Shih et al. 2003). UI is also associated with caregiver morbidity, stress and depression (Ory et al. 1986, Yu et al. 1991, Ouslander \& Schnelle 1995) which can result in their inability to care and older people being admitted into a care home (Thom et al. 1997).

This paper reports on a systematic review of systematic reviews that have synthesized the evidence on the management of UI and promotion of continence in older people in care home populations using conservative/ behavioural techniques, such as, bladder training or toileting programmes, which form the basis of nursing care. It provides a broad, comprehensive synthesis of empirical evidence from systematic reviews and provides a narrative synthesis of reviews, interventions, descriptive studies, outcomes and review quality. Umbrella reviews or systematic reviews of reviews are emerging form of evidence synthesis (Smith et al. 2011, Cooper \& Koenka 2012) and this is the first such review on this topic. As such, this review can inform future directions for research, 
guidelines for practice and having implications for clinical practice at a local level.

\section{Background}

Clinical guidelines (Fantl et al. 1996, Button et al. 1998, NICE 2006, 2007), international consultations (Abrams et al. 2009) and Cochrane reviews (Eustice et al. 2000, Ostaszkiewicz et al. 2004a,b, Wallace et al. 2004) have synthesized evidence to inform clinical practice for the management of UI, although none are specific to older people in care homes where prevalence is highest. In the USA, the US Department of Health \& Human Sciences, Medicare and Medicaid Services mandates that each nursing home resident who has UI is 'identified and assessed and provided with appropriate treatment and services to achieve or maintain as much normal urinary function as possible' (DHHS 2005, F315). Other countries, such as Australia (ACF 2013), Canada (CCF 2013) and England (NICE 2006, 2007) also recommend nationally good clinical practice, guidelines and standards, for managing UI although a recent repeat national audit in England found they are not always adhered to in nursing or care homes; they are not mandatory, financial penalties are not incurred and so reimbursements are not affected (Wagg et al. 2005, 2007, Potter et al. 2007).

Institutional settings in the community that provide care for older people (generic term care homes) include nursing homes (providing nursing care), residential homes (providing mainly social care which also includes managing UI) or mixed/aged care homes (providing both nursing and social care). The majority of research into the management of UI has been undertaken in hospital or community populations (Abrams et al. 2009). Research on the management of UI in older people in care homes is available. Studies have largely been undertaken in the USA with designated research teams (e.g. Schnelle et al. 1989, Colling et al. 1992, Ouslander et al. 1995), although there is an emerging body of evidence from other countries of care homes staff managing UI (Tobin \& Brocklehurst 1986, Jilek 1993, Sgadari et al. 1997, Gaitsgori et al. 1998, Aslan et al. 2008, Sackley et al. 2008, Tanaka et al. 2009).

Behavioural interventions (bladder training (BT), prompted voiding (PV), habit retraining (HR), timed voiding (TV)) are commonly used to manage UI in care home residents, with 20-40\% estimated to receive them (Brandeis et al. 1997, Jumadilova et al. 2005). A metastudy of four Cochrane reviews (BT, PV, HR, TV) concluded a need to revisit theory, definitions and contents underpinning each of these technologies as there was some overlap in operational components with no overall consensus (Roe et al. 2007a,b). The maintenance of continence in older people in care homes has barely featured in empirical studies.

\section{The review}

\section{Aim}

To inform future research, clinical guidelines and current practice by the synthesis of evidence from systematic reviews on the management of UI and promotion of continence using conservative/behavioural approaches in older people in care homes.

\section{Objectives}

To undertake a narrative synthesis of evidence from systematic reviews on the effects of intervention studies using conservative/behavioural approaches to manage UI or promote continence and their outcomes on continence status or associated factors in older people in care homes.

To undertake a narrative synthesis of evidence from systematic reviews that have included descriptive studies using conservative/behavioural approaches related to the management of urinary incontinence or promotion of continence and their outcomes on continence status or associated factors in older people in care homes.

\section{Design}

Systematic review of systematic reviews. The protocol was registered with the Faculty Research Ethics Committee.

\section{Methods}

In reviews of reviews, quality appraisal, data extraction and evidence synthesis take place at the level of the review rather than the individual study. The review methods adhered to the PICOS Framework (Robinson et al. 2011) and PRISMA Statement for reporting systematic reviews (Liberati et al. 2009, Moher et al. 2009) and guided by Smith et al. (2011) and Ryan et al. (2012).

\section{Search methods}

Five most relevant databases MEDLINE, CINAHL, The Cochrane Library (including CRD-Centre for Reviews and Dissemination) and PROSPERO (the international register of systematic reviews), were searched from their inception to December 2012 to locate systematic reviews published in 
English. Searches were updated in September 2013 and no date restrictions were applied. Reference sections of yielded reviews were also searched

\section{Search strategy}

A copy of the search strategies for MEDLINE and CINAHL are available (see Table S1 in the online version). The MEDLINE expanded search strings from the Cochrane Incontinence Review Group for UI were used and included all empirical research designs (Grant et al. 2006). The search strings were modified to enhance their selectiveness for older people and care homes and to exclude studies involving surgical or pharmacological interventions alone. The PICOS Framework (Robinson et al. 2011) was used to inform the search strategy and the inclusion and exclusion criteria.

\section{Inclusion criteria}

Systematic reviews of empirical studies of the management of UI, promotion or maintenance of continence in older people aged 65 years and over in care homes were located. Reviews of conservative/behavioural approaches with incontinence specified or defined were included (Figure 1).

\section{Exclusion criteria}

Reviews of surgical studies or pharmaceutical interventions alone were excluded as the focus was on conservative behavioural approaches which are care practices predominantly undertaken by nurses or care assistants in care homes (Figure 2).

\section{Search outcome}

Electronic searches located 40 reviews of which 33 were not relevant and were excluded. Hand searching located a further two potential reviews. A total of nine reviews were independently screened by three reviewers and agreement reached to include or exclude. Five were included as they met al.1 of the inclusion criteria while four were excluded. Three Cochrane reviews did not fulfil all of the inclusion criteria, but had been identified in previous reviews and used to locate studies that fulfilled the criteria from their included and excluded studies tables (Eustice et al. 2000, Ostaszkiewicz et al. 2004a,b). One review was excluded because it did not fulfil all of the inclusion criteria and met one of more of the exclusion criteria, although for this review it was used to check original relevant studies had been included in the reviews included in this review (Shamliyan et al. 2007). See PRISMA flowchart (Figure 3) and logic decision tree explanation and list of excluded reviews (Figure S1).

\section{Quality appraisal}

Quality of included systematic reviews was assessed independently by two reviewers using AMSTAR (Shea et al.

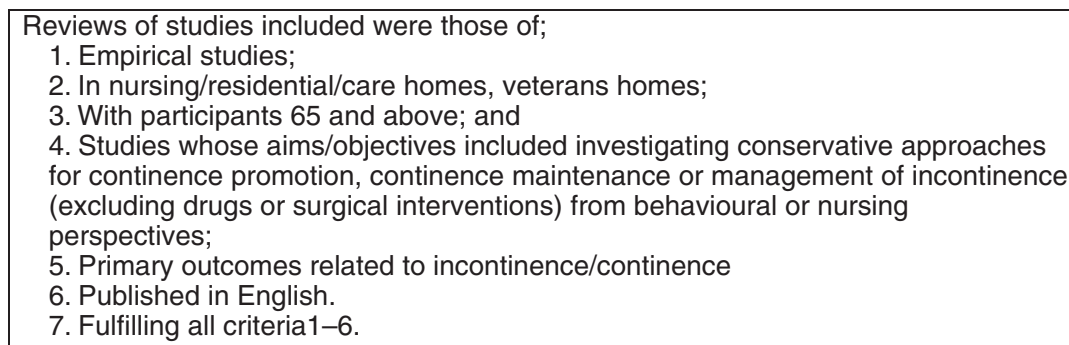

Figure 1 Inclusion criteria for reviews.

\footnotetext{
Reviews were excluded if;

1. Studies or articles were not empirical;

2. If their studies included adults below 65 years;

3. Involved drugs only and/or surgery;

4. Studies aims/objectives were not related to conservative approaches for continence maintenance, continence promotion or management of incontinence; 5. Primary outcomes were not related to incontinence/continence;

6 . Were conducted in hospital, participants' home, rehabilitation facilities, 'care in the community', 'step-down' beds or community settings other than care homes, nursing homes, residential homes.

7. Studies where participants only attend the nursing homes, residential homes, care homes or assisted living facilities on a day case basis and were not residents; 8. Not published in English.

9. If any one of the above occurred
}

Figure 2 Exclusion criteria for reviews. 
Figure 3 PRISMA flow diagram for included reviews.

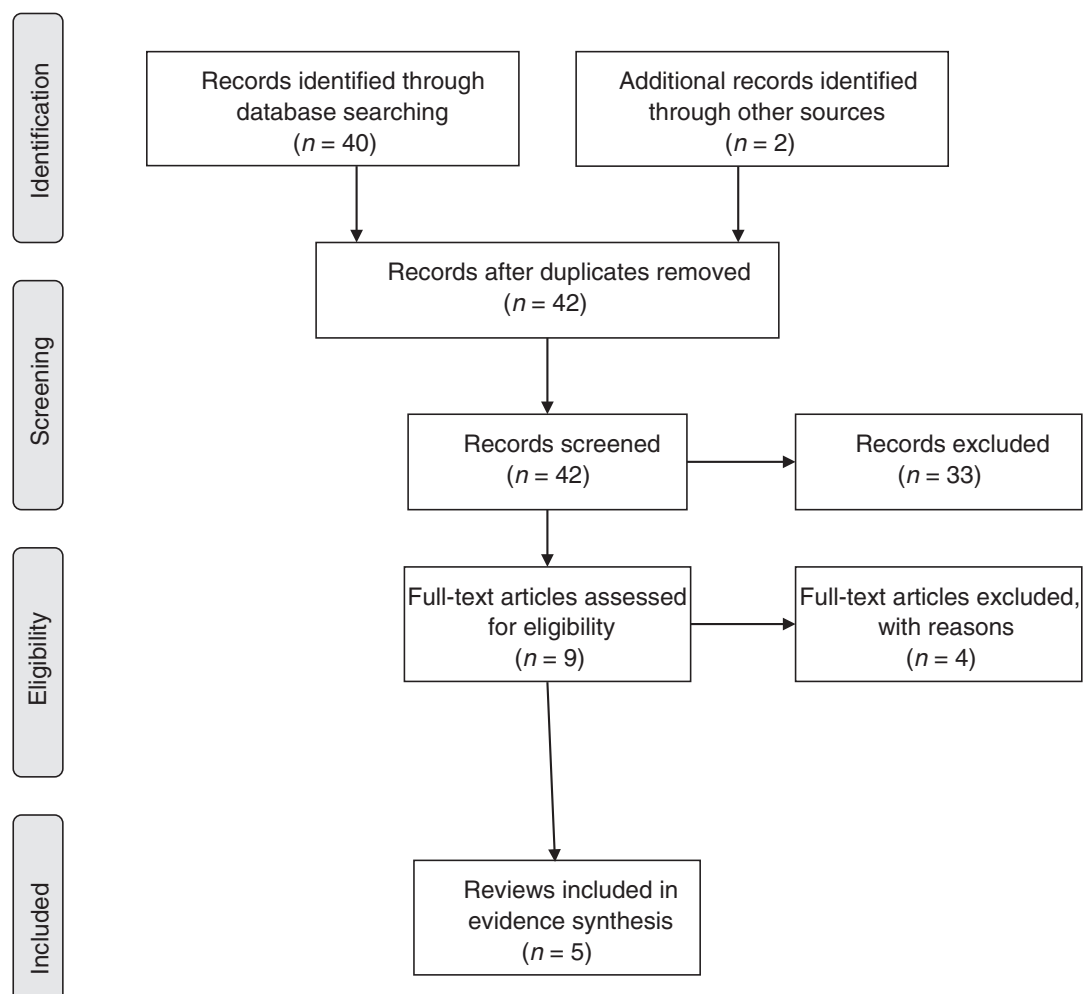

2007), with consensus for the final score. AMSTAR scores avoidance of bias in review methods against 11 distinct criteria. Each criteria is scored yes (clearly done), no (clearly not done), cannot answer or not applicable. The higher the score the higher the quality rating ( 3 or lower - low quality; 4-7 medium quality; $8-11$ high quality). No reviews were excluded on the basis of the quality score.

\section{Data extraction}

A bespoke data extraction form, agreed by the reviewers, was developed based on the PRISMA Statement (Liberati et al. 2009) and systematic review of systematic reviews methodology (Smith et al. 2011, Ryan et al. 2012). Electronic versions were used independently by the three reviewers to extract data. The information was checked by a second reviewer for accuracy and agreement reached for all included reviews. Two reviewers had oversight of all included reviews and data extraction, one of whom was an experienced reviewer completely independent of the published reviews and who provided additional quality assurance. Data were extracted on scope/aim of the review, search strategy, number of studies, settings, participants; age and gender, study designs, inclusion/exclusion criteria, outcomes, quality appraisal of studies and review.

\section{Data synthesis}

Due to heterogeneity of reviews, it was not possible to perform meta-analyses. In this review, we extracted data for the primary outcomes, as reported by reviewers, as numerical data and descriptive summaries to allow consistent reporting across the reviews. The research designs included intervention and descriptive studies with primary outcomes related to continence or factors associated with incontinence. Two reviewers analysed and summarized the information from the included reviews and reported them as narratives to allow the identification of broad conclusions in and across the reviews and reach consensus. Summary tables as used by Smith et al. (2011) and Ryan et al. (2012) have been used to present results in a structured format to enhance textual commentary.

\section{Results}

Five relevant systematic reviews that met the inclusion criteria were included (See Figure 3 flow diagram outlining the selection process). Justification for their inclusion and reasons for the exclusion of four other potential reviews along with their details are reported in Figure S1. The review by Fink et al. (2008) was led from the USA while 
the other four parallel systematic reviews were led from England (Roe et al. 2011, 2013, Flanagan et al. 2012, 2014).

\section{Aims and scope of the reviews}

The aim of the review by Fink et al. (2008) was to determine efficacy and safety of treatments for nursing home residents with UI. They included behavioural and pharmacological studies $(n=14)$, of which 10 studies were relevant to this review of reviews synthesizing evidence on behavioural/conservative approaches predominantly delivered by qualified nurses, nursing assistants or carers for the management of incontinence or promotion of continence (Table 1). The four other reviews were parallel reviews that aimed to review published empirical studies using behavioural/conservative techniques to manage UI, promote continence or maintain continence in older people in care home populations. Flanagan et al. (2012, 2014) review intervention studies while Roe et al. (2011, 2013) review descriptive studies with UI as the primary outcome or factors associated with care the primary outcome. Due to heterogeneity of the studies narrative syntheses have been undertaken (Table 1).

\section{Study characteristics and populations}

The reviews reported on 72 studies relevant to this review of reviews (data adjusted to account for the duplication of six included studies across two reviews). Country of origin of studies were reported in the four reviews with the majority $(76 \%, 52)$ conducted in the USA and the remainder from 10 countries plus one international study that reported on seven countries (Sgadari et al. 1997). Four reviews reported dates of studies ranging from 1980-2009; with most being published in the 1990s (30), slightly fewer in the 2000s (26) and least in the 1980s (12) (Table 1). A total of 1930 care homes were included with data reported from over half a million residents $(535,178)$ and a minority of staff or family (Table 1). Total number of residents with UI reported in reviews ranged from 701-444,429 with mean ages ranging from 73.9-88.7 years. Residents with UI in the care homes (defined in the reviews and including nursing homes, residential homes, dual registered homes, aged care, Veterans' homes and assisted living) tended to be older with the vast majority of residents being women (71\%, 380,684) (Table 2). The majority of studies recruited or reported on residents with UI only $(51,67 \%)$ with the remainder reporting on residents with UI with or without concomitant FI.

\section{Methodological components of included reviews}

All reviews included in this review adhered to the PRISMA statement (Liberati et al. 2009) and included a PRISMA flowchart (Moher et al. 2009). Criteria for the inclusion and exclusion of empirical studies were specified in each review along with their literature search strategy and dates of searching (Tables 1 and S3). Studies searched for and located were all empirical and published in English and each review included details of their literature search strategies (Table 1). There was overlap between the Fink et al. (2008) and Flanagan et al. (2012) studies with the same 6 RCTs included in both reviews. The overall and adjusted totals for studies and residents are reported (Tables 1 and $2)$. The majority of individual studies included their inclusion criteria $(53,74 \%)$ while only half cited their exclusion criteria $(38,53 \%)$ (Table 3). Nearly, two-third of studies were interventions $(46,64 \%)$ with the remainder descriptive studies $(26,36 \%)$. Only four studies were economic evaluations. Virtually all studies were quantitative or mixed methods with only three studies solely using qualitative designs. Of the evaluated interventions, half were RCTs or quasi RCTs $(23,50 \%)$ with around a third uncontrolled studies $(17,37 \%)$ (Table S3).

Only a very small minority of studies included power calculations or justification of sample $(16,22 \%$; intervention studies: three power calculations and eight purposive sampling; descriptive: 5). Eight intervention studies (17\%) included intention to treat analysis while slightly more studies, irrespective of design, reported loss to follow-up along with reasons, although still a minority $(14,19 \%)$. No longterm follow-up of participants or residents were reported in the intervention studies, while a minority of descriptive studies reported long-term follow-up $(7,26 \%$ with three following up to 1 year) (Table S3).

\section{Quality of included reviews}

All five reviews scored nine of 11 on AMSTAR indicating they were of high quality and is likely to have minimal bias in their design and conduct. Sources of funding were recorded in all reviews and no declarations of interest that posed a conflict were also included in four reviews.

\section{Quality of included studies}

The reviews included methods of assessing quality of included studies although the quality scores were not reported in one review (Fink et al. 2008) (Table S3). The other reviews used standard checklists for quantitative or 


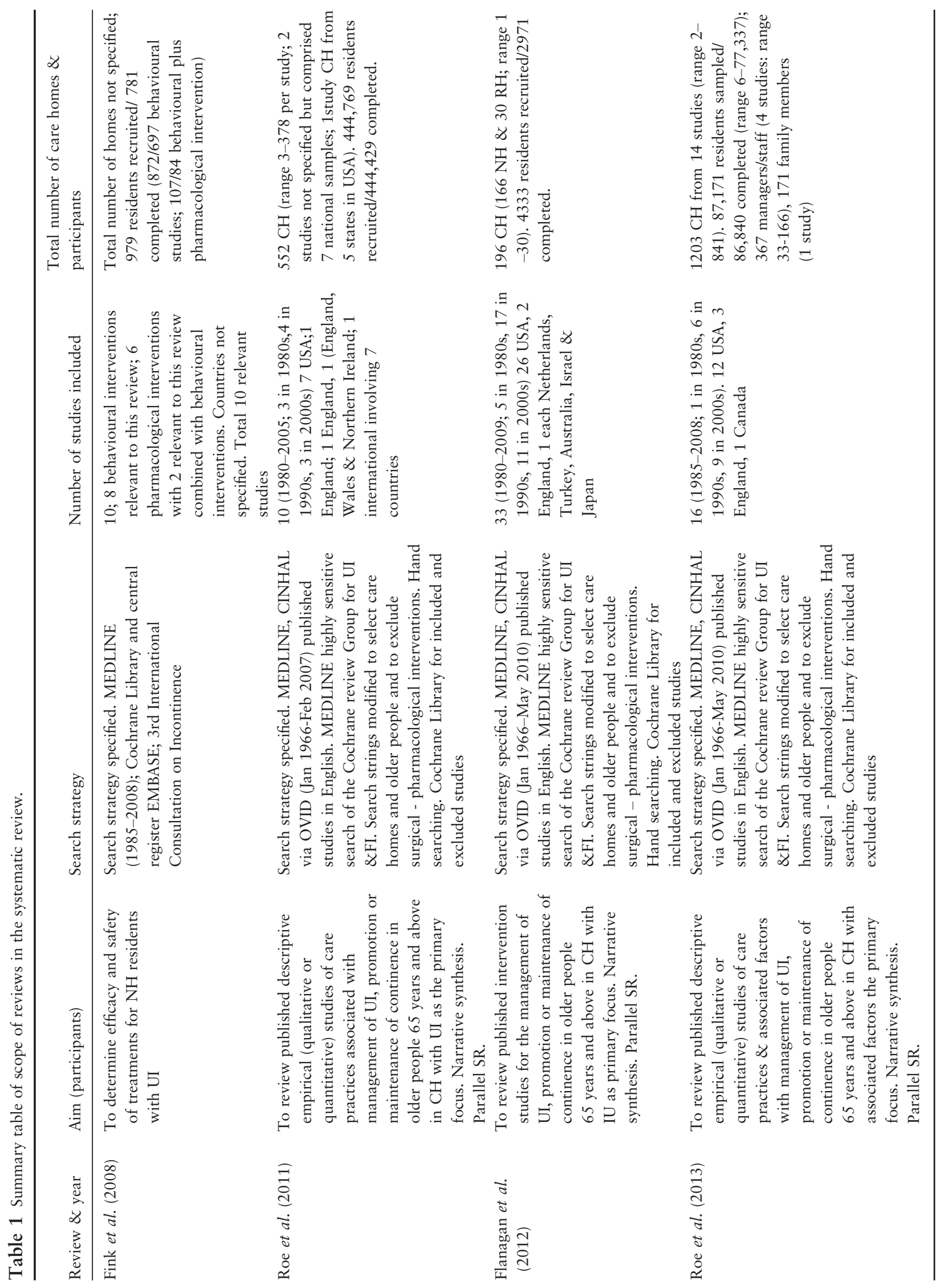


B. Roe et al.

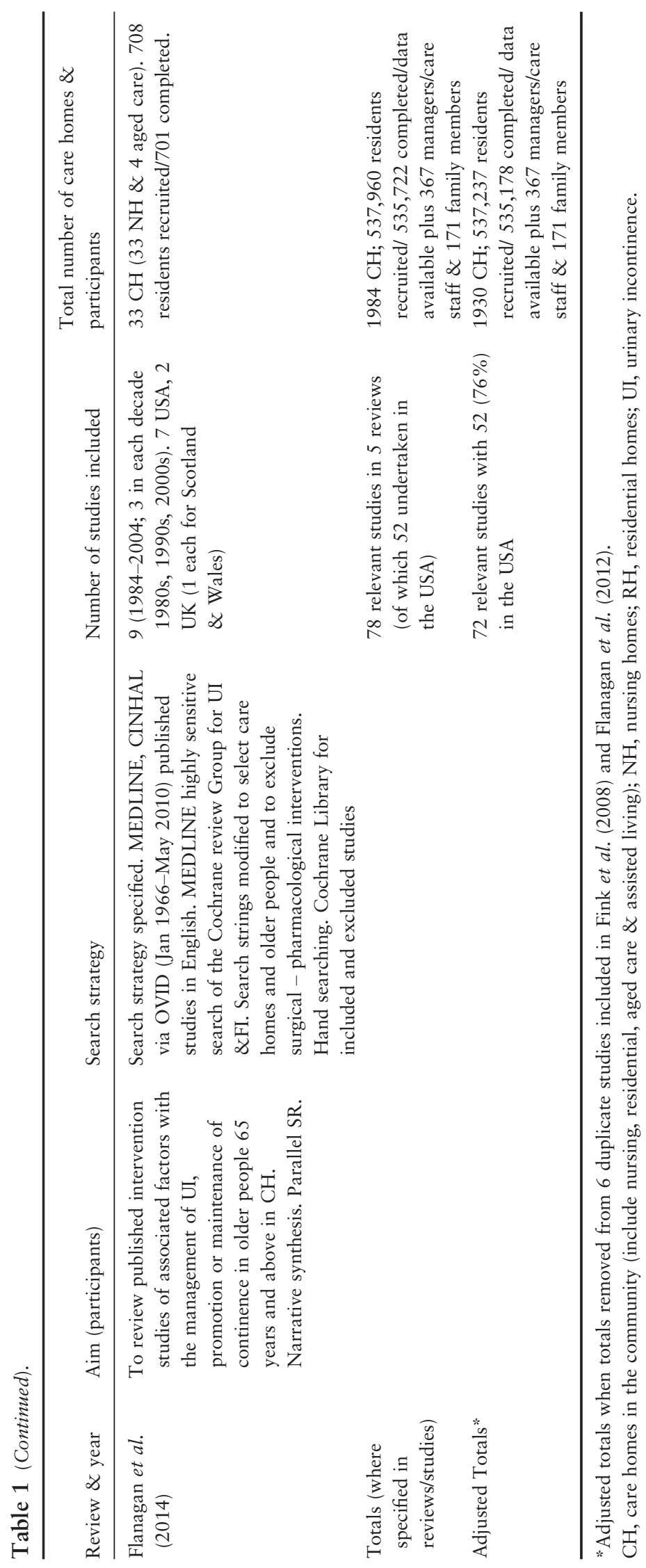


Table 2 Summary table of resident participants, mean age range \& gender where reported in studies in systematic review of reviews.

\begin{tabular}{|c|c|c|c|}
\hline Review \& year & $\begin{array}{l}\text { Residents/participants } \\
\text { completed }\end{array}$ & $\begin{array}{l}\text { Range of mean age } \\
\text { in years }\end{array}$ & Gender \\
\hline Fink et al. (2008) & 781 & $\begin{array}{l}73 \cdot 9-88 \cdot 6 \text { - mean age } \\
\text { reported in } 9 \text { studies }\end{array}$ & $\begin{array}{l}635(59 \%) \text { women; } 444(41 \%) \text { men, reported in } 9 \text { studies } \\
\text { (7 behavioural \& } 2 \text { relevant combined behavioural and } \\
\text { pharmacological) }\end{array}$ \\
\hline Roe et al. (2011) & 444,429 & $\begin{array}{l}77-88.7 \text { - mean age } \\
\text { reported in } 7 \text { studies }\end{array}$ & $\begin{array}{l}321,073(72 \%) \text { women; } 122,021(28 \%) \text { men, reported } \\
\text { in } 7 \text { studies }\end{array}$ \\
\hline $\begin{array}{l}\text { Flanagan et al. } \\
\text { (2012) }\end{array}$ & 2971 & $\begin{array}{l}78-91 \cdot 3 \text { - mean age } \\
\text { reported } 31 \text { studies }\end{array}$ & $\begin{array}{l}2019(77 \%) \text { women; } 615(23 \%) \text { men, reported in } 26 \\
\text { studies }\end{array}$ \\
\hline Roe et al. (2013) & 86,840 & $\begin{array}{l}81 \cdot 5-86 \cdot 5 \text { - mean age } \\
\text { reported by } 6 \text { studies }\end{array}$ & $\begin{array}{l}56,992(66 \%) \text { women; } 29,848(34 \%) \text { men, reported } \\
\text { in } 8 \text { studies }\end{array}$ \\
\hline $\begin{array}{l}\text { Flanagan et al. } \\
\text { (2014) }\end{array}$ & 701 & $\begin{array}{l}81 \cdot 5-85 \cdot 6 \text { - mean age } \\
\text { reported by } 6 \text { studies }\end{array}$ & $250(72 \%)$ women; $99(28 \%)$ men, reported in 4 studies \\
\hline Total & 535,722 & $73 \cdot 9-88 \cdot 7$ & $\begin{array}{l}380,969(71 \%) \text { women; } 153,027(29 \%) \text { men, reported in } \\
54 \text { studies }\end{array}$ \\
\hline Adjusted Totals* & 535,178 & $73 \cdot 9-88 \cdot 7$ & $\begin{array}{l}380,684(71 \%) \text { women; } 152,956(29 \%) \text { men reported in } \\
47 \text { studies }\end{array}$ \\
\hline
\end{tabular}

*Adjusted totals when totals removed from 6 duplicate studies included in Fink et al. (2008) and Flanagan et al. (2012).

qualitative studies, used by Shaw et al. (2009, p. 9-11, appendixes 1 and 2) adapted from Downs and Black (1998) and Kmet et al. (2004) and made relevant to each review. The quality of intervention studies was appraised using The Cochrane Incontinence Review Group criteria for the assessment of quality of randomized/quasi-randomized controlled trials (Grant et al. 2006). In the Fink et al. (2008), review quality of studies was assessed for random allocation concealment ( 1 poor to 3 best quality), blinding of participants and assessors, intention to treat analysis, loss to follow-up with reasons, but scores for studies were not reported although narrative descriptions were. Studies were not excluded on the basis of their quality score in the reviews.

Where quality ratings were reported, only 20 (43\%) trials could be assessed for random allocation concealment with two being adequate $(\mathrm{A})$ and the remainder unclear $(\mathrm{B}, 9)$ or not concealed $(\mathrm{C}, 9)$ with the majority at risk of allocation bias.

Quality scores ranged from 22-100\% with 15 (58\%) of descriptive studies and $17(37 \%)$ of intervention studies scoring $75 \%$ or above. Less than half the included studies were rated of higher quality $(32,44 \%)$. More recently published studies tended to be rated higher quality. Only nine descriptive studies reported on reliability and validity of methods and their rigour (Table S3).

\section{Main findings and conclusion of reviews}

The main findings, outcomes and conclusions from the reviews are presented here and in summary Table 3. More detailed summary results (intervention and comparison, outcome, number of studies and results) for included studies for each review are available in a Table (S2 available online).

\section{Intervention studies}

Three reviews synthesized evidence on intervention studies (Fink et al. 2008, Flanagan et al. 2012, 2014, Tables S2, S3). Fink et al. (2008) included RCTs of behavioural $(n=8)$ and pharmacological interventions $(n=6)$, two of the latter were relevant and included in this review of reviews as they combined behavioural interventions with drugs or placebo. Two reviews of interventions where UI was the primary outcome measure (Fink et al. 2008, Flanagan et al. 2012) were trials of toileting programmes; the majority PV vs. usual care or PV combined with exercise and mobility training, or PV plus a drug vs PV plus a placebo. Two trials compared other toileting programmes; patterned urge response toileting vs usual care and toilet skill training vs. usual care with improvements in toileting, UI and wet checks for those receiving the intervention. PV alone or PV with exercise are associated with modest short term improvement in UI. The evidence on use of drugs in conjunction with PV is limited and no evidence showing a benefit with oestrogen. Long-term follow-up, QOL measures and economic evaluation are required. Meta-analysis was not possible due to the heterogeneity of studies. There was some evidence to indicate labour costs of toileting and number of staff required were higher than laundry costs based on estimates in two studies. One study indicated 
Table 3 Summary table of main findings and conclusions reported in systematic review of reviews.

\begin{tabular}{|c|c|}
\hline Review \& year & Summary of findings/conclusion \\
\hline Fink et al. (2008) & $\begin{array}{l}\text { All trials included residents with UI. Trials of toileting programmes } \\
\text { vs usual care (5RCTs) consistently found an improvement in } \\
\text { continence. PV alone or PV with exercise were associated with } \\
\text { modest short term improvement in daytime UI. } \\
\text { No supporting evidence for independent effects with exercise alone. } \\
\text { Oxybutynin may provide some benefit with PV. No role for } \\
\text { oestrogen in UI treatment. Long term clinical trials of PV alone, PV } \\
\text { with exercise should be conducted with targeted NH residents with } \\
\text { UI. These trials should include measures of UI, patient QOL and } \\
\text { cost outcomes. Trials of drugs plus toileting are too few to make } \\
\text { any recommendations }\end{array}$ \\
\hline
\end{tabular}

Roe et al. (2011) 7 studies included residents with UI only and 3 studies residents with UI or UI and FI. Studies involved mainly women with mean age $>80$ years. Prevalence of UI higher than FI, more women affected than men. Prevalence of UI is higher in institutional settings.

Studies demonstrate there are improvements in the implementation of care for managing incontinence in care home residents. Combined evidence suggests that conservative approaches for managing incontinence and promoting continence using pads and toileting are the most frequent for residents. Use of incontinence pads and toileting (to include BT, scheduled toileting and prompted voiding) were the most prevalent forms of management and feature of documented policies.

PV with physical exercise for residents shows some evidence of effectiveness (Schnelle et al. 2002). Other forms of management, such as, pelvic floor muscle exercises, drugs, catheters or penile sheaths featured less frequently.

Improvements in assessment of incontinence and documenting practice have been identified over the last three decades, although there are variations between and within countries. Wagg (2005) found treatment goals were documented for $54 \%$ of residents with $76 \%$ of homes reporting they would involve residents in choice of incontinence products. Only $2 \%$ of family members were reported as being involved in decisions for the management of incontinence by Watson (2003).

Two studies assessed whether the current management of incontinence should be changed and identified this was the case for only a minority of residents (Peet 1996, Watson 2003). Watson (2003) concluded that the national AHRQ guideline (Fantl et al. 1996) had been under-utilized in care homes although its use was feasible

Flanagan et al. 26 studies UI only; 7 studies UI and/or FI. A large proportion of (2012)

\section{Comments}

All trials of behavioural interventions relevant to the SR of SRs, only 2 trials of PV plus drug vs PV plus placebo (Ouslander 1995, 2001) from pharmacological interventions were relevant and were also included in the review by Flanagan et al. 2012.

Trials of toileting programmes vs usual care (5 RCTs) consistently found an improvement in continence.

Operational definitions and content of toileting programmes have not been included in studies and may not reflect contemporary developments in behavioural techniques and interventions. There is a lack of longitudinal studies incorporating documentary review and observed practice for these populations. Further research is warranted to determine outcomes and improvements in continence status. Studies targeted at maintaining continence in residents who are continent should also be undertaken. Involving residents or family members in decisions for managing incontinence is poorly reported and should be more widely practised.

Studies on maintaining continence and identification of components of toileting programmes that make them successful including full economic evaluation are also indicated

Meta-analysis not possible due to heterogeneity studies. Labour costs to implement toileting higher than laundry cost inferred by one study but no economic data included. Another study specified 1 Nursing assistant was required for 5 residents to implement toileting. Based on mean time to implement care 20.7 minutes, estimating a ratio of 1:5; 1 study indicated 2 hourly toileting did not confer benefit to UI compared to 3 hourly and increases workload. Economic implications estimated or inferred in three studies related to primary outcome of UI. 
Table 3 (Continued).

\begin{tabular}{|c|c|c|}
\hline Review \& year & Summary of findings/conclusion & Comments \\
\hline Roe et al. (2013) & 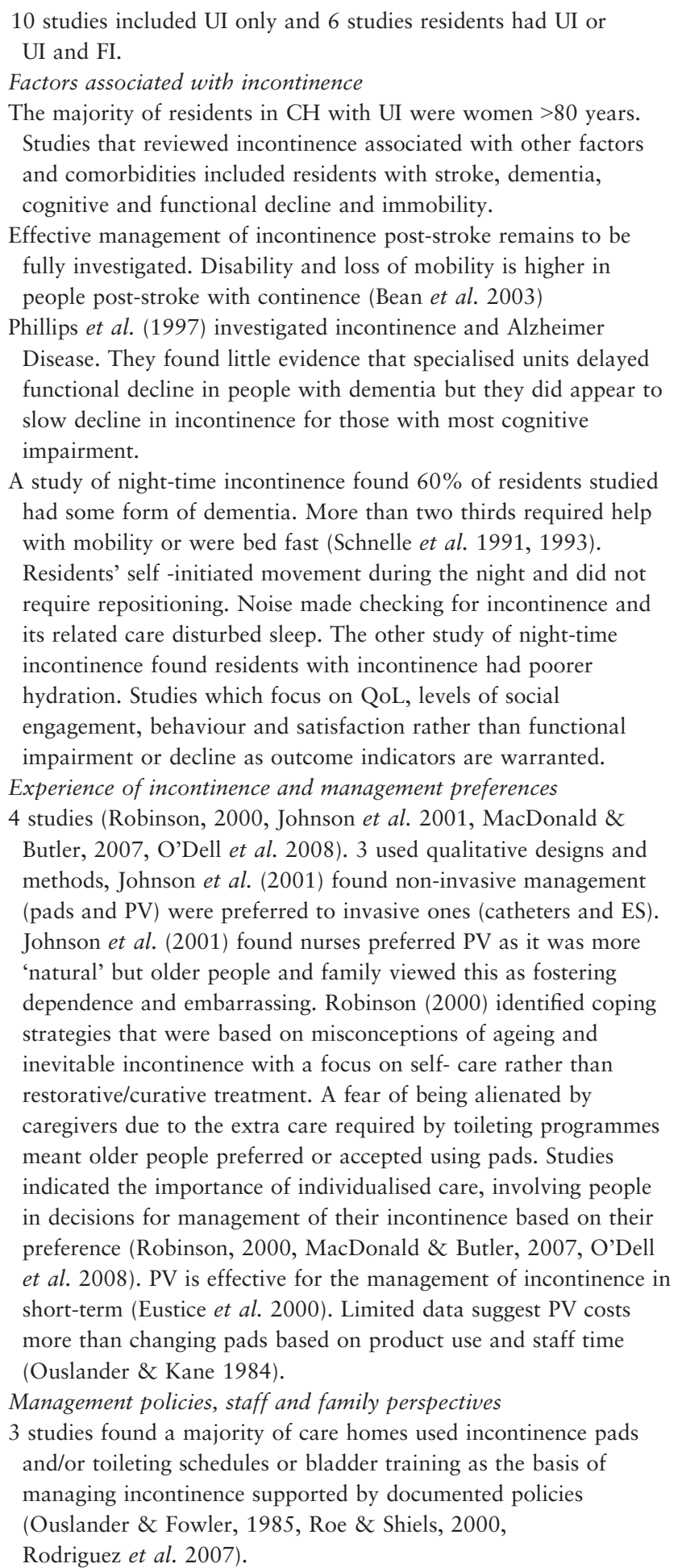 & $\begin{array}{l}\text { All but four studies were undertaken in the USA, } \\
\text { with most studies published this century. All of } \\
\text { the studies investigated factors associated with } \\
\text { the management of UI with or without FI. No } \\
\text { study looked at maintenance of continence. } \\
\text { Quantitative or mixed methods formed the basis } \\
\text { of the studies with only three studies } \\
\text { incorporating qualitative methods }\end{array}$ \\
\hline
\end{tabular}


Table 3 (Continued).

\begin{tabular}{l} 
Review \& year $\quad$ Summary of findings/conclusion \\
\hline What the toileting programmes or bladder training comprised was \\
not always made explicit; only specified by a minority. The \\
Veterans populations in the care homes had high use of indwelling \\
catheters which denotes homes with predominantly male residents \\
and date of study (Ouslander \& Fowler, 1985). Toileting \\
programmes or non-invasive methods for management are \\
recommended due to the high infection rates and morbidity \\
associated with indwelling catheters (Hu et al. 1990, Durrant \& \\
Snape, 2003).Studies of management of incontinence at the level \\
individuals and systems within care homes taking account of \\
organisations, culture, policies, staffing, attitudes, demands, \\
workloads, priorities and finance are warranted. Implementing \\
interventions requires complex organisational change. \\
Incontinence is associated with other factors (causal or \\
consequence) that need to be considered when planning and \\
managing care for individuals, developing and designing systems \\
of care within homes and future research. \\
These associated factors mean that management of incontinence or \\
promotion of continence involves complex combined interventions \\
that need to be targeted at different levels; individuals, staff and \\
organisations. \\
Non-invasive methods, such as toileting and use of pads, are \\
common approaches to managing incontinence in residents in care \\
homes. Older people and their family should be involved with \\
decisions for their care, management of incontinence, goals and \\
outcomes. Preventive studies that maintain continence for these \\
populations are required. \\
4 UI
\end{tabular}

(2014) specified but PV described and usually undertaken for UI. So inferred/proxy and by outcome data reported.

Schnelle et al. 1991 suggest with education PV is transferable to other NH populations. Few studies have looked at the cost effectiveness of interventions for managing incontinence. Three studies agreed the costs of implementing toileting programmes are higher than incontinence products alone and one study showed toileting was more expensive than 2 of the incontinence products tested. However, using the toilet is normal behaviour can preserve dignity and can avoid skin problems due to incontinence. Toileting can be more expensive where residents are physically dependent and require staff to help them. Toileting programmes are beneficial in reducing incontinence and maintained over time. Longer term implementation and follow up are required. Maximising quality of care and resident's wellbeing are priorities. Managing incontinence and skin care are components of this.

The studies did show some evidence that use of $\mathrm{pH}$ cleansers with or without barrier cream were beneficial compared to soap and water in relation to skin integrity and less time consuming. UI\&FI can cause excoriated skin, dermatitis and pressure ulcers which are painful and impair function and QOL. Good skin care is fundamental to QOL of care and managing incontinence.
Comments

The review was of factors associated with incontinence as primary outcomes and included economic factors of managing incontinence, skin integrity and skin care protocols, staff quality control processes and adherence to toileting protocols, as well as promoting continence through hydration, prompted voiding, toileting assistance and changing pad/ linen through 'rounding.' The studies did show benefits for all approaches. However, studies were few in number per topic. The skin care studies had small sample sizes and all studies had no long term follow up. Few relied on nursing assistants to undertake the interventions. Future studies aimed at implementation of interventions for promoting continence, maintaining continence and managing incontinence in older people in care homes with $\mathrm{NH}$ staff that include outcomes for UI/FI, associated factors and effectiveness with economic evaluation are warranted. 
Table 3 (Continued).

\begin{tabular}{|c|c|c|}
\hline Review \& year & Summary of findings/conclusion & Comments \\
\hline & $\begin{array}{l}\text { NA were successful in implementing toileting programmes, PV and } \\
\text { can benefit reducing rates of UI. PV plus exercise showed benefits } \\
\text { with residents' mobility during the intervention and over time. } \\
\text { Further trials with blinding for exercise and toileting programmes } \\
\text { are warranted over longer time and those residents who benefit } \\
\text { most identified. } \\
\text { One study, Schnelle et al. 1991, highlighted the importance of } \\
\text { adopting a staff quality control process for care for residents who } \\
\text { are bed bound and requiring incontinence pad/linen change more } \\
\text { than toileting programmes. They measured adherence to toileting } \\
\text { programmes (PV) by staff and conclude that quality care requires } \\
\text { staff quality control processes requiring protocols, staff training, } \\
\text { adherence and quality checks, data analysis to ensure processes of } \\
\text { care are implemented and can be by nursing staff not research } \\
\text { staff. These findings are from one study. } \\
\text { One study of 'care rounding' every } 1.5 \text { hours to promote } \\
\text { hydration, offering drinks, offering prompts and assistance for } \\
\text { toileting, skin cleansing and pad changing found improved } \\
\text { hydration of residents and a reduction in incontinence and soiling/ } \\
\text { increased continence (Spangler et al. 1984). Ensuring adequate } \\
\text { hydration of older residents is an important associated factor } \\
\text { related to incontinence and an indicator of quality care }\end{array}$ & \\
\hline
\end{tabular}

BT, bladder training; ES, electrical stimulation; FI, faecal incontinence; PV, prompted voiding; UI, urinary incontinence.

there were no benefits to UI in two hourly compared with three hourly toileting.

The other review on interventions investigated factors associated with managing incontinence or promoting continence (Flanagan et al. 2014). These studies included skin care, staff adherence to toileting protocols and the promotion of continence through hydration, PV, toileting assistance and changing pads/linen through 'rounding'. These studies did show benefits through active management however they were very few on each topic; only three RCTs included economic evaluation and as such provide limited evidence (Table 3).

\section{Descriptive studies}

Two reviews included descriptive studies, one with UI as the primary outcome (Roe et al. 2011) and the other factors associated with managing UI the primary outcome (Roe et al. 2013) (Table 3). The reviews identified toileting programmes and incontinence pads were the main forms of management and featured in documented policies. Assessment of UI and documented care plans with treatment goals that residents are involved in still require emphasis but there has been improvement over time evidenced in two studies and use of guidelines to inform practice are feasible. Older people and their family pre- ferred non-invasive management and wished to be involved in decisions about their care although one study identified residents' fear of alienating staff due to extra care required for toileting and accepted or preferred using incontinence pads. These studies demonstrate context for interventions used to manage UI and promote continence but again are few in number and evidence is limited from small single studies. The international study across seven countries found variation between and in countries for the management of UI in these populations (Sgadari et al. 1997). Economic evaluation featured in two studies and no studies investigated how continence can be maintained in these populations (Table 3).

\section{Outcomes}

For interventions with UI as the primary outcome, measurements reported included incontinence episodes (day only or day and night), wet checks, appropriate and/or independent toileting and time taken toileting to determine intervention effectiveness (Tables 3 and S2). PV only and PV plus exercise significantly decreased UI episodes, increased appropriate toileting and toilet requests in the short term up to 6/8 weeks. Only one trial found benefit in supplementing PV with oxybutynin. No significant improvement in UI outcomes were found for the three trials that investigated indi- 
vidualized toileting plus facilitated exercise or mobility or PV plus oestrogen/progesterone (Tables 3 and S2).

In trials that investigated factors associated with the management of UI outcomes included staff costs, laundry changes and costs, pad changes $(n=3)$, skin integrity, incontinence dermatitis $(n=3)$, exercise $(n=1)$, appropriate toileting, wet checks, linen check and change $(n=1)$ pad weight and measure of dehydration $(n=1)$ (Tables 3 and S2). Economic evaluations found PV or toileting programmes increased staff workload with costs higher than laundry costs and pad changes increased vs. usual care, although one study reported a significant increased saving per patient in laundry costs. Skin care studies found $\mathrm{pH}$ cleansers were better than soap and water in maintaining skin integrity but sample sizes were small. Where PV was undertaken with exercise there was an increase in daily exercise, mean sit to stand per day and significant improvement in exercise endurance vs. PV only $(n=1)$. Staff quality control and adherence to PV and linen check protocols $(n=1)$ found no significant differences in appropriate toileting, wetness or average volume of incontinence and concluded that staff training in adherence to toileting interventions is required. One further study that measured dehydration and weight of pads following 'rounding' which included prompting for drinks, toileting, pad/linen change vs. usual care had positive effects on hydration and continence in non-ambulatory residents. However it should be noted these studies are few, have small samples but do infer benefit.

Descriptive studies provide context and breadth of factors associated with managing UI for older people in care homes: including prevalence and incidence, management, economic evaluation, comorbidities, experience of UI, management preference, policies, staff perspectives or methodology (Tables 3 and S2). Studies were diverse and varied with no uniform approach. Outcomes included prevalence, incidence, assessment and documentation, policies, management techniques, estimated costs, use of pads/ catheters, toileting, PV, assessment and diagnosis of UI, feasibility of using guidelines (Tables 3 and S2). They describe the full range of factors that need to be considered when managing UI in these populations and interventions did not take them into account in their design or methods.

\section{Discussion}

This review has provided a narrative synthesis of evidence on conservative - behavioural approaches to the management of UI and promotion of continence in older people in care homes. Including relevant reviews of intervention and descriptive studies ensured a breadth and context of evidence with a wide lens on the range of diverse studies. The main interventions are toileting, PV in particular with or without exercise/mobility and use of incontinence pads. The reviews found evidence of benefit for PV (with and without exercise/mobility) in reducing UI, improving request and appropriate toileting in the short term with increased costs for staff time and workload. This is in keeping with the Cochrane review on PV (Eustice et al. 2000) conclusion that long-term follow-up studies are warranted; our review concurs with this.

There was limited evidence from studies in reviews on individualized toileting or toilet training skills. How these compare to TV is unclear but again from Cochrane reviews of other toileting interventions, HR (Ostaszkiewicz et al. 2004a) and TV (Ostaszkiewicz et al. 2004b), there is insufficient evidence to guide practice. TV is the mainstay of clinical practice and regular toileting is frequently undertaken, 2 hourly. The study by Schnelle et al. (2002) found no benefit of 2 hourly PV vs. 3 hourly PV, although this evidence is only from one study.

A metastudy of the four Cochrane reviews of behavioural interventions BT, PV, HR and TV found overlap in operational terminology, although not always stated in studies nor the content of each technique comprehensively described (Roe et al. 2007a,b). The metastudy concluded that the theory and content underpinning these toileting programmes should be revisited in future studies and evidence on all toileting programmes is being reviewed and synthesized (Ostaszkiewicz et al. 2013). Interventions with supported facilitation, longterm follow-up or economic evaluation were very limited and should be incorporated into future studies.

Studies on the maintenance of continence for people in care homes are lacking. The outcomes of interventions at the level of studies were variable and did not include QoL measures as standard. Future interventions should adhere to established standardized outcome measures with Cochrane reviews on incontinence a potential template. Future studies could then be included in future updates of relevant Cochrane systematic reviews.

What usual care constitutes was never fully described. Usual care could involve some form of toileting or prompting and potentially confounding the toileting intervention being tested. If people who are non-ambulatory are just been washed and changed as usual care, this may not constitute ethical practice as it is normal or usual for people to use the toilet, with assistance if necessary. Even if commodes, bedpans or urinals are used it is essential that a person's dignity and privacy are assured (BGS 2007, NT Clinical Update 2013). 
An early intervention study reviewed by Flanagan et al. (2014; see Spangler et al. 1984) investigated hourly 'rounding' to promote and offer hydration, toileting, cleansing and changing where required by non-ambulatory residents and had positive benefits for continence and significant improvement for hydration. 'Intentional rounding' was introduced in the USA by the Studer Group (2007) and has subsequently been implemented in hospitals by NHS England (Fitzsimons et al. 2011, Bartley 2012, Levenson 2013) following concerns about poor standards of care. Quality of nursing care is of international importance and has been under increased scrutiny in England following a recent public inquiry, particularly for older people requiring care (Francis 2013). 'Intentional rounding' aims to ensure that all patients are seen regularly by staff on a rote basis to meet essential needs relating to fluid intake, skin care and toileting (Fitzsimons et al. 2011, Bartley 2012), similar to the study above by Spangler et al. (1984) in care homes. The advent of the nursing process, care plans and individualized care saw the demise of 'rounds' although 'intentional rounding' is not incompatible with providing individual care. However, 'intentional rounding' is not without critics and some view it as a retrograde step (Levenson 2013, p6, Snelling 2013).

A recent phase II feasibility trial of managing UI poststroke in hospitals has suggested that 'intentional rounding' could have influenced usual care when compared with a systematic voiding programme (of BT or PV) (Thomas et al. in press) denoting it was having an effect, potentially procedural confounding the intervention under study. To protect and promote high standards of individual person-centred care and ensure quality it may be essential to rediscover 'embodied practice' which forms the 'heart' of nursing care (Draper 2014). Meeting essential needs relating to hydration, skin care and toileting constitute 'embodied practice' and the basis of managing UI, promoting continence and maintaining continence in older people in care homes, who constitute potentially vulnerable populations unable to meet basic human needs.

Trials that incorporate the role of evidence, context and facilitation are now emerging (Rycroft-Malone et al. 2013), specifically for the management of UI in care home populations (Seers et al. 2012) and for people post stroke in hospital (Thomas et al. 2011, 2014). A majority of interventions in the reviews pre-date The CONSORT Statement (2010) and a lack of standardized reporting meant complete information was not always available. Standardized reporting for publication of future studies will assist with evidence synthesis. Reviews noted that more recently published studies were of higher quality.
Descriptive studies included a broad range of relevant factors and aspects of care which warrant inclusion in future research. Similarly many of the studies pre-date the COREQ publication standard for qualitative research (Tong et al. 2007). Due to the diverse range and few studies, often with small samples, the findings are indicators of what practice and future studies need to consider. There was limited evidence on people's experience and preference for management of UI, family and staff perspectives. Involving people in decision-making for care is essential. There was limited evidence of the benefit of $\mathrm{pH}$ skin cleansers in maintaining skin health over and above soap and water. Documented policies for managing UI in care home populations are increasingly available with the need for assessment and diagnosis of UI. There was evidence that assessment and diagnosis to inform care was being undertaken but not for a majority of residents (Resnick et al. 1996, Wagg et al. 2005, 2007) although feasible (Resnick et al. 1996); this varied in care home populations across countries (Sgadari et al. 1997). Regular national audits however have shown improvements over time (Wagg et al. 2005, 2007, Roe et al. 2013). Guidelines and evidence for managing UI are available (Fantl et al. 1996, Button et al. 1998) and their use feasible (Watson 2004).

Whole system approaches or soft system analysis (Checkland \& Poulter 2006, Michie et al. 2011) that incorporate organization and service delivery, such as care home culture and policies, staffing levels, staff attitudes and resident preferences have not really featured in intervention or observational studies, but are warranted. Implementation studies using whole systems approaches for the management of UI in care homes with long-term follow-up are required. Future trials which adhere to frameworks for complex interventions that include standardized outcomes (MRC 2000, 2008), with embedded qualitative descriptive, mixed methods approaches investigating whole systems including context, process and facilitation of implementation, with short- and long-term follow-up of outcomes are also warranted.

\section{Limitations of the review}

A strength and limitation of the review was its broad but inclusive focus on reviews of studies investigating the management of UI and promotion of continence in older people in care home populations. The most relevant databases were searched as evidence exists that multiple database searching provides little gain (Gorecki et al. 2010, Beyer \& Wright undated). A narrative synthesis was only possible due to heterogeneity between and in reviews and studies. The majority of studies were undertaken in the USA and care home popu- 
lations there are not comparable with care home populations in other countries as size, culture, funding and staffing vary.

\section{Conclusion}

Toileting programmes, PV in particular with or without exercise and use of incontinence pads, for managing UI in older people in care homes is effective in the short term, reliant on staff adherence and resource. Interventions with long-term follow-up are warranted but designs need to account for usual care also involving some form of toileting. Descriptive mixed methods studies should be embedded into interventions that allow measures of context and explain the impact of other factors have on implementation. Where possible standard outcomes, QoL measures and economic evaluation should be included with designs adhering to complex intervention frameworks and reporting meeting international standards for publication. Studies maintaining continence for older people in care homes are also indicated. Implications for practice are that assessment and diagnosis of incontinence, treatment of remedial comorbidities, individual toileting, use of incontinence pads, attention to hydration, skin care and maintaining optimum mobility and exercise are also essential for this vulnerable population. Involving older people and family as partners in their care is paramount. All of which are not only indicators of quality care but also still core components of nursing practice.

\section{Funding Information}

None declared.

\section{Conflict of interest}

No conflict of interest was declared by the authors in relation to the study itself. Note that Brenda Roe is a JAN editor but, in line with usual practice, this paper was subjected to double-blind peer review and was edited by another editor.

\section{Author contributions}

All authors agreed on the final version and meet at least one of the following criteria [recommended by the IC-MJE (http://www.icjme.org/ethical_1author.html)]:

- substantial contributions to conception and design, acquisition of data, or analysis and interpretation of data;

- drafting the article or revising it critically for important intellectual conent.

\section{Supporting Information}

Additional Supporting Information may be found in the online version of this article at the publishers web-site.

\section{References}

Abrams P., Cardozo L., Khoury S. \& Wein A. (2009) Fourth International Consultation on Incontinence. Health Publications Ltd, Plymouth, MN.

ACF (2013) Australian Continence Foundation. Retrieved from www.continence.org.au/pages/professioanls.html on 30 December 2013.

Aggazzotti G., Pesce F., Grassi D., Fantuzzi G., Righi E., De Vita D., Santacroce S. \& Artibani W. (2000) Prevalence of urinary incontinence among institutionalized patients: a cross-sectional epidemiological study in a mid-sized city in northern Italy. Urology 56, 245.

Aslan E., Komurcu N., Beji N.K. \& Yalcin O. (2008) Bladder training and Kegel exercises for women with urinary complaints living in a rest home. Gerontology 54, 224-231.

Bartley A. (2012) The Hospital Pathways Project. Making it Happen: Intentional Rounding. The Kings Fund Point of Care and The Health Foundation, London.

Berlowitz D.R., Brandeis G.H., Anderson J. \& Brand H.K. (1997) Predictors of pressure ulcer healing among long-term care residents. Journal of the American Geriatrics Society 45(1), 30-34.

Beyer F. \& Wright K. (undated) Comprehensive Searching for Systematic Reviews: A Comparison of Database Performance. Centre for Reviews and Dissemination, University of York, York. Retrieved from http://www.york.ac.uk/inst/crd/Posters/ Comprehensive_searching_for_systematic_reviews.pdf on 29 October 2014.

BGS (2007) Behind Closed Doors. British Geriatrics Society, London. Retrieved from www.bgs.org.uk/campaigns/dignity.htm on 22 March 2014

Borrie M.J. \& Davidson H.A. (1992) Incontinence in institutions: costs and contributing factors. Canadian Medical Association Journal 147(3), 322-328.

Brandeis G.H., Baumann M.M., Hossain M., Morris J.N. \& Resnick N.M. (1997) The prevalence of potentially remediable urinary incontinence in frail older people: a study using the Minimum Data Set. Journal of the American Geriatrics Society 45(2), 179-184.

Brown J., Vittinghoff E., Feng Lin M., Nyberg L., Kusek J.W. \& Kanaya A.M. (2006) Prevalence and risk factors for urinary incontinence in women with type 2 diabetes and impaired fasting glucose: findings from the National Health and Nutrition Examination Survey (NHANES) 2001-2002. Diabetes Care 29 (6), 1307-1312.

Button D., Roe B., Webb C., Frith T., Colin Thome D. \& Gardner L. (1998) Consensus guidelines for the management of continence by health care teams: development, implementation and evaluation. Journal of Advanced Nursing 27, 91-99.

CCF (2013) Canadian Continence Foundation. Guidelines. Retrieved from www.canadiancontinence.com/english/healthprofs/guidelines/html on 30 December 2013. 
Checkland P. \& Poulter J. (2006) Learning for Action: A Short Definitive Account of Soft Systems Methodology and its Use for Practitioners, Teachers and Students. Wiley, Chichester.

Colling J., Ouslander J., Hadley B.J., Eisch J. \& Campbell E. (1992) The effect of Patterned Urge Response Toileting (PURT) on urinary incontinence among nursing home residents. Journal of the American Geriatrics Society 40(2), 135-141.

Cooper H. \& Koenka A.C. (2012) The overview of reviews: Unique challenges and opportunities when research syntheses are the principal elements of new integrative scholarship. American Psychologist 67, 446-462.

DH (2000) Good Practice in Continence Service. Department of Health, London. Retrieved from http://www.continencefound ation.org.uk/campaigns/goodpracticecontinence.pdf on 28 March 2008.

DHHS (2005) CMS Manual System Department of Health \& Human Services (DHHS) Pub. 100-07 State Operations Provider Certification, Centers for Medicare \& Medicaid Services (CMS), Transmittal 8 Date: JUNE 28, 2005. F315.

Diokno A.C., Brock B.M., Brown M.B. \& Herzog A.P. (1986) Prevalence of urinary incontinence and other urological symptoms in non-institutionalzed elders. Journal of Urology 136, 1022.

Downs S.H. \& Black N. (1998) The feasibility of creating a checklist for the assessment of the methodological quality both of randomised and non-randomised studies of health care interventions. Journal of Epidemiology and Community Health $52,377-384$.

Draper J. (2014) Embodied practice: rediscovering the 'heart' of nursing. Journal of Advanced Nursing 70 (10), 2235-2244 (early online 27 MAR 2014, DOI: 10.1111/jan.12406).

DuBeau C.E., Simon S.E. \& Morris J.N. (2006) The Effect of urinary incontinence on quality of life in older nursing home residents. Journal of the American Geriatrics Society 54(9), 1325-1333.

DuBeau C.E., Kuchel G.A., Johnson T., Palmer M.H. \& Wagg A. (2009) Incontinence in the frail elderly. In Incontinence, 4th edn (Abrams P., Cardozo L., Khoury S. \& Wein A., eds), Health Publications Ltd, Paris, 961 p. Retrieved from www.ncbi.nlm.nih.gov/pubmed/2002502 on 16 July 2012.

Dumoulin C., Korner-Bitensky N. \& Tannenbaum C. (2007) Urinary incontinence after stroke: identification, assessment and intervention by rehabilitation professionals in Canada. Stroke 38 (10), 2745-2751.

Eustice S., Roe B. \& Paterson J. (2000) Prompted voiding for the management of urinary incontinence in adults. Cochrane Database of Systematic Reviews (2), CD002113. doi: 10.1002/ 14651858.CD002113

Fantl J.A., Newman D.K., Colling J., Delancey J.O.L., Keeys C., Loughery R., McDowell B.J., Norton P., Ouslander J., Schnelle H.J., Staskin D., Tries J., Urich V., Vitousek S.H., Weiss B.D. \& Whitmore K. (1996) Urinary Incontinence in Adults: Acute and Chronic Management. Clinical Practice Guideline. No.2 update. Agency for Health Care Quality and Research, Rockville, MD.

Fink H.A., Taylor B.C., Tacklind J.W., Rutks I.R. \& Wilt T.J. (2008) Treatment interventions in nursing home residents with urinary incontinence: A systematic review of randomized trials. Mayo Clinic Proceedings 83(12), 1332-1343.
Fitzsimons B., Bartley A. \& Cornwell J. (2011) Intentional rounding: its role in supporting essential care. Nursing Times 107(27), 18-19.

Flanagan L., Roe B., Jack B., Barrett J., Chung A., Shaw C. \& Williams K. (2012) A systematic review of care intervention studies for the management of incontinence and promotion of continence in older people in care homes with urinary incontinence as the primary focus. Geriatrics and Gerontology International 12(4), 600-611.

Flanagan L., Roe B., Jack B., Barrett J., Chung A., Shaw C. \& Williams K. (2014) Systematic review of care intervention studies that investigated associated factors with the management of incontinence and promotion of continence in older people in care homes. Journal of Advanced Nursing 70(3), 476-496. First published 25 July 2013. doi: 10.1111/jan122220

Foley A., Loharuka S., Barrett J.A., Mathews R., Williams K., McGrother C. \& Roe B. (2012) Association between the geriatric giants of urinary incontinence and falls in older people using the data from the Leicestershire MRC Incontinence study. Age and Ageing 41(1), 35-39. doi:10.1093/ageing/afr125.

Francis R. (2013) Report of the Mid-Staffordshire NHS Foundation Trust Public Inquiry. The Stationery Office, London.

Gaitsgori Y., Gruenwald I., Zarmi S. \& Michalak R. (1998) Individual timed voiding as a long term treatment modality for demented patients in nursing homes. Neurourology and Urodynamics 17(4), 323-332. Abstract from 28th Annual Meeting of the International Continence Society (ICS) Jerusalem, Israel.

Gorecki C.A., Brown J.M., Briggs M. \& Nixon J. (2010) Evaluation of five search strategies in retrieving qualitative patient-reported electronic data on the impact of pressure ulcers on quality of life. Journal of Advanced Nursing 66(3), 645-652.

Grant A.M., Cody D.J., Glazener C.M.A., Hay-Smith J., Herbison P., Lapitan M.C., Moore K.N., Norton C., Wallace S.A. \& Wilson P.D. (2006) Incontinence Group. About Cochrane Collaboration (Cochrane Review Groups (CRGS) 2000). Issue 4. Retrieved from http://www.incontinence.cochrane.org on 11 October 2010.

Grant R.L., Drennan V.M., Rait G., Peterson I. \& Illiffe S. (2013) First diagnosis and management of incontinence in older people with and without dementia in primary care: a cohort study using The Health Improvement Network primary care database. PLoS Medicine August 27, 2013. doi: 10.1371/journalpmed.1001505

Hu T.W., Igou J.F., Kaltreider L., Yu L.C., Rohner T.J., Dennis P.J., Craighead E., Hadley E.C. \& Ory M.G. (1989) A Clinical trial of behavioural therapy to reduce urinary incontinence in nursing homes. Journal of the American Geriatrics Society 261 (18), 2656-2662.

Hu T.W., Kaltreider L., Igou J.F., Yu L.C. \& Rohner T.J. (1990) Cost effectiveness of training incontinent elderly in nursing homes: A randomized clinical trial. Health Services Research. 25 (3), 455-477.

Jilek R. (1993) Elderly toileting: is two hourly too often? Nursing Standard 7(47), 25-26.

Jorgensen L., Engstad T. \& Jacobsen B.K. (2005) Self-reported urinary incontinence in non-institutionalized long term stroke survivors. A population based survey. Archives of Physical Medicine \& Rehabilitation 86(3), 416-420. 
Jumadilova Z., Zyczynski T., Paul B. \& Narayanan S. (2005) Urinary incontinence in the nursing home: resident characteristics and prevalence of drug treatment. American Journal of Managed Care 11(4 suppl), S112-S120.

Kmet L.M., Lee R.C. \& Cook L.S. (2004) Standard Quality Assessment Criteria for Evaluating Primary Research Papers from a Variety of Fields. HTA Initiative \#13. The Alberta Heritage Foundation for Medical Research, Edmonton, AB. Retrieved from http://www.ahfmr.ab.ca on 11 October 2010.

Kron M., Loy S., Sturn E., Nikolaus T. \& Becker C. (2003) Risk indicators for falls in institutionalized frail elderly. American Journal of Epidemiology 158(7), 645-653.

Levenson R. (2013) A report on the implementation of intentional rounding at demonstrator sites for the nursing and quality care forum. The Nursing and Quality Care Forum, August 2013, vs. 3.0. NHS England, UK. Retrieved from www.6cs.england.nhs.uk/dl/cv_content/81294 on 22 March 2014.

Liberati A., Altman D.G., Tetzlaff J., Mulrow C., Gotzsche P.C., Ioannidis J.P.A., Clarke M., Devereaux P.J., Kleijinene J. \& Moher D. (2009) The PRISMA Statement for reporting systematic reviews and meta-analyses of studies that evaluate health care interventions: explanation and elaboration. PLoS Medicine. 6(7), e1000100.

McGrother C.M., Donaldson M., Wagg M., Matharu G., Williams K.S. \& Watson J.M. (2003) Healthcare Needs Assessment: The Epidemiologically Based Needs Assessment Reviews. Radcliffe Medical Press Ltd, Abingdon.

Michie S., van Stralen M.M. \& West R. (2011) The behaviour change wheel: A new method for characterising and designing behaviour change interventions. Implementation Science 6, 42.

Moher D., Liberati A., Tetzlaff J., Altman D.G. \& The PRISMA Group (2009) Preferred reporting items for systematic reviews and meta-analyses: The PRISMA statement. PloS Medicine 6, e100009.

MRC (2000) A Framework for the Development and Evaluation of RCTs for Complex Interventions to Improve Health. Medical Research Council, London.

MRC (2008) Developing and Evaluating Complex Interventions: New Guidance. Medical Research Council, London.

NICE (2006) Urinary Incontinence: The Management of Urinary Incontinence in Women. NICE Clinical Guideline 40. National Institute of Health and Clinical Excellence, London.

NICE (2007) Faecal Incontinence: NICE Clinical Guideline 49. National Institute of Health and Clinical Excellence, London.

NT Clinical Update (2013) Ensuring patient dignity when accessing and using toilets. Retrieved from http://www.nursingtimes.net/ Journals/2013/02/08/b/m/w/070424Ensuring-patient-dignitywhen-accessing-and-using-toilets.pdf on 22 March 2014.

Ory M.G., Wyman J.F. \& Yu L. (1986) Psychosocial factors in urinary incontinence. Clinics in Geriatric Medicine 2(4), 657671.

Ostaszkiewicz J., Chestney T. \& Roe B. (2004a) Habit retraining for the management of urinary incontinence in adults. Cochrane Database of Systematic Reviews (2), CD002801. doi: 10.1002/ 14651858.CD002801.pub2.

Ostaszkiewicz J., Johnston L. \& Roe B. (2004b) Timed voiding for the management of urinary incontinence in adults. Cochrane
Database of Systematic Reviews (1), CD002802. doi: 10.1002/ 14651858.CD002802.pub2.

Ostaszkiewicz J., Eustice S., Roe B., Thomas L.H., French B., Islam T., O’Connell B. \& Cody J.D. (2013) Toileting assistance programmes for the management of urinary incontinence in adults (Protocol). Cochrane Database of Systematic Reviews (6), CD010589. doi: 10.1002/14651858.CD010589.

Ouslander J.G. \& Schnelle J.F. (1995) Incontinence in the nursing home. Annals of Internal Medicine 122, 438.

Ouslander J.G., Schnelle J.T., Uman G., Fingold S., Nigam J., Tuico E. \& Bates-Jenson B. (1995) Predictors of successful prompted voiding among incontinent nursing home residents. Journal of American Medical Association 273(17), 1366-1370.

Palmer M.H. (2009) Urinary incontinence and overactive bladder in patients with heart failure. Journal of Urology 182(1), 196-202.

Potter J., Peel P., Mian S., Lowe D., Irwin P., Pearson M. \& Wagg A. (2007) National audit of continence care for older people: management of faecal incontinence. Age and Ageing 36(3), 268-273.

Resnick N.M., Brandeis G.H., Baumann M.M. \& Morris J.N. (1996) Evaluating a national assessment for urinary incontinence in nursing home residents: reliability of the minimum data set and validity of the residents assessment. Protocol. Neurourology and Urodynamics 15, 583-589.

Richardson J.P. \& Hriez L. (1995) Risk factors for the development of bacteremia in nursing home patients. Archives of Family Medicine 4(9), 785-789.

Robinson K.A., Saldanha I.J. \& McKoy N.A. (2011) Framework for Determining Research Gaps During Systematic Reviews Systematic Reviews. Methods Future Research Needs Report No2. AHRQ Publication No 11-EHC043-EF. Agency for Healthcare Research and Quality, Rockville, MD.

Roe B., Milne J., Ostaszkiewicz J. \& Wallace S. (2007a) Systematic reviews of bladder training and voiding programmes in adults: A synopsis of findings on theory and methods using Metastudy techniques. Journal of Advanced Nursing 57(1), 314.

Roe B., Ostaszkiewicz J., Milne J. \& Wallace S. (2007b) Systematic reviews of bladder training and voiding programmes in adults: A synopsis of findings from data analysis and outcomes using Metastudy techniques. Journal of Advanced Nursing 57(1), 15-31.

Roe B., Flanagan L., Jack B., Barrett J., Chung A., Shaw C. \& Williams K. (2011) Systematic review of the management of incontinence and promotion of continence in older people in care homes. Descriptive studies with urinary incontinence as primary focus. Journal of Advanced Nursing 67(2), 228-250.

Roe B., Flanagan L., Jack B., Shaw C., Williams K., Chung A. \& Barrett J. (2013) Systematic review of descriptive studies that investigated associated factors with the management of incontinence in older people in care homes. International Journal of Older People Nursing 8(1), 29-49. Online early - 19 Dec 2011. doi: 10.1111/j.1748-3743.2011.00300.x

Ryan R., Santesso N., Hill S., Lowe D., Kaufman C. \& Grimshaw J. (2012) Consumer-oriented interventions for evidence-based prescribing and medicine use: an overview of systematic reviews. 
The Cochrane Library. Cochrane Database of Systematic Reviews 2011 (5), CD007768.

Rycroft-Malone J., Seers K., Chandler J., Hawks C.A., Crichton N., Allen C., Bullock I. \& Strunin L. (2013) The role of evidence, context and facilitation in implementation trial: implications for the development of the PARIHS framework. Implementation Science 8, 28.

Sackley C.M., Rodriguez N.A., van den Berg M., Badger F., Wright C., Besemer J., van Reeuwijk K.T.V. \& van Wely L. (2008) A phase II exploratory cluster randomised controlled trial of a group mobility training and staff education intervention to promote urinary continence in UK care homes. Clinical Rehabilitation 22, 714-721.

Schnelle J.F., Traughber B., Sowell V.A., Newman D.R., Petrilli C.O. \& Ory M. (1989) Prompted voiding treatment of urinary incontinence in nursing home patients: a behavior management approach for nursing home staff. Journal of the American Geriatrics Society 37(11), 1051-1057.

Schnelle J.F., Alessi C.A., Simmons S.F., Samarrai N.R., Beck J.C. \& Ouslander J.G. (2002) Translating clinical research into practice: a randomized controlled trial of exercise and incontinence care with nursing home residents. Journal of the American Geriatrics Society 50(9), 1476-1483.

Seers K., Cox K., Crichton N.J., Tudor Edwards R., Eldh A.C., Esabrooks C.A., Harvey G., Hawkes C., Kitson A., Linck P., McCarthy G., McCormack B., Mockford C., Rycroft-Malone J., Titchen A. \& Wallin L. (2012) FIRE (Facilitating Implementation of Research Evidence): a study protocol. Implementation Science 7, 25.

Sgadari A., Topinkova E., Bjornson J. \& Bernabei R. (1997) Urinary Incontinence in nursing home residents: A cross national comparison. Age and Ageing 26(Suppl 2), 49-54.

Shamliyan T., Wyman J., Bliss D.Z., Kane R.L. \& Wilt T.J. (2007) Prevention of Urinary and Fecal Incontinence in Adults. Evidence Report/Technology Assessment No 161, AHRQ Publication No 08-E003. Agency for Healthcare Research and Quality, Rockville, MD.

Shaw C., McNamara R., Abrams K., Cannings-John R., Hood K., Longo M., Myles S., O’Mahoney S., Roe B. \& Williams K. (2009) Systematic review of respite care in the frail elderly. Health Technology Assessment 13, 20. Retrieved from http:// www.ha.ac.uk on 11 October 2010.

Shea B.J., Grimshaw J.M., Wells G.A., Boers M., Andersson N., Hamel C., Porter A.C., Tugwell P., Moher D. \& Bouter L.M. (2007) Development of AMSTAR: a measurement toll to assess the methodological quality of systematic reviews. BMC Medical Research Methodology 7, 10. doi: 10.1186/1471-22887-10.

Shih Y.C., Hatzema A.G. \& Tolleson-Rinehart S. (2003) Labor costs associated with incontinence in long-term care facilities. Urology 62(3), 442-446.

Smith V., Devane D., Begley C.M. \& Clarke M. (2011) Methodology in conducting a systematic review of systematic reviews of healthcare interventions. BMC Medical Research Methodology 11, 15. Retrieved from www.biomedcentral.com/ 1471-2288/11/15 on 01 September 2013.

Snelling P. (2013) Intentional rounding: a critique of the evidence. Nursing Times 109(20), 19-21.
Spangler P., Risley T. \& Bilyew D. (1984) The management of dehydration and incontinence in nonambulatory geriatric patients. Journal of Applied Behaviour Analysis 17, 397-401.

Spector W.D. (1994) Correlates of pressure sores in nursing homes: evidence from the National Expenditure Survey. Journal of Investigative Dermatology 102(6), 42S-45S.

Studer Group (2007) Best Practices. Sacred Heart Hospital, Pensacola, Florida. Hourly Rounding Supplement. Studer Group, Gulf Breeze, FL.

Tanaka Y., Nagata K., Tanaka T., Kuwano K., Endo H., Otani T., Nakazawa M. \& Koyama H. (2009) Can an individualized and comprehensive care strategy improve Urinary Incontinence (UI) among nursing home residents? Archives of Gerontology and Geriatrics 49, 278-283.

The CONSORT Statement (2010) CONSORT Transparent reporting of trials. Retrieved from http://www.consortstatement.org/consort-statement/ on 24 March 2014.

Thom D.H., Haan M.N. \& Van den Eeden S.K. (1997) Medically recognized urinary incontinence and risks of hospitalization, nursing home admission and mortality. Age and Ageing 26, 367.

Thomas L.H., Watkins C.L., French B., Sutton C.J., Forshaw D., Cheater F., Roe B., Leathley M., Burton C., McColl E. \& Booth J. (2011) ICONS: Identifying Continence OptioNs after Stroke: a randomised trial. BMC Trials 12, 131.

Thomas L.H., French B., Burton C.R., Sutton C., Forshaw D., Dickinson H., Leathley M., Britt D., Roe B., Chester F.M., Booth J. \& Watkins C.L. on behalf of the ICONS Project Team, the ICONS Patient, Public and Carer Involvement Groups (2014) Evaluating a systematic voiding programme for patients with urinary incontinence after stroke in secondary care using soft systems analysis and Normalisation Process Theory: Findings from the ICONS case study phase. International Journal of Nursing Studies 51(10), 1308-1320. doi:10.1016/ j.ijnurstu.2014.02.009. E online 21 March 2014.

Thomas L.H., French B., Sutton C.J., Forshaw D., Leathely M.J., Burton C.R., Roe B., Cheater F.C., Booth J., McColl E., Carter B., Walker A., Brittain K., Whiteley G., Rodgers H., Barrett J. \& Watkins C.L. (in press) ICONS: Identifying continence options after stroker: An evidence synthesis, case study and exploratory cluster randomised controlled trial of the introduction of a systematic voiding programme for patients with urinary incontinence after stroke in secondary care. Final Report. NIHR Journals Library: Programme Grants for Applied Research.

Tobin G.W. \& Brocklehurst J.C. (1986) The management of urinary incontinence in local authority residential homes for the elderly. Age and Ageing 15(5), 292-298.

Tong A., Sainsbury P. \& Craig J. (2007) Consolidated criteria for reporting qualitative research (COREQ): a 32 item checklist for interviews and focus groups. International Journal of Quality in Health Care 19(6), 349-357.

Wagg A., Mian S., Lowe D., Potter J. \& Pearson M. (2005) National audit of continence care for older people: Results of a pilot study. Journal of Evaluation in Clinical Practice 11(6), 525-532.

Wagg A., Potter J., Peel P., Irwin P., Lowe D. \& Pearson M. (2007) National audit of continence care for older people: management of urinary incontinence. Age and Ageing 37(1), 39-44. 
Wallace S.A., Roe B., Williams K. \& Palmer M. (2004) Bladder training for urinary incontinence in adults. Cochrane Database of Systematic Reviews (1), CD001308. doi: 10.1002/ 14651858.CD001308.pub2

Watson N.M. (2004) Advancing quality of urinary incontinence evaluation and treatment in nursing homes through translational research. Worldviews on Evidence-based Nursing $1, \mathrm{~s} 21-\mathrm{s} 25$.
Williams M.P., Srikanth V., Bird M. \& Thrift A.G. (2012) Urinary symptoms and natural history of urinary continence after first ever stroke: a longitudinal population-based study. Age and Ageing 41(3), 371-376.

Yu L.C., Johnson K., Kaltreider D.L., Hu T.W., Brannon D. \& Ory M. (1991) Urinary incontinence: nursing home staff reaction towards residents. Journal of Gerontological Nursing 17(11), $34-41$.

The Journal of Advanced Nursing (JAN) is an international, peer-reviewed, scientific journal. JAN contributes to the advancement of evidence-based nursing, midwifery and health care by disseminating high quality research and scholarship of contemporary relevance and with potential to advance knowledge for practice, education, management or policy. JAN publishes research reviews, original research reports and methodological and theoretical papers.

For further information, please visit JAN on the Wiley Online Library website: www.wileyonlinelibrary.com/journal/jan

$\underline{\text { Reasons to publish your work in } J A N \text { : }}$

- High-impact forum: the world's most cited nursing journal, with an Impact Factor of 1.527 - ranked 14/101 in the 2012 ISI Journal Citation Reports (C) (Nursing (Social Science)).

- Most read nursing journal in the world: over 3 million articles downloaded online per year and accessible in over 10,000 libraries worldwide (including over 3,500 in developing countries with free or low cost access).

- Fast and easy online submission: online submission at http://mc.manuscriptcentral.com/jan.

- Positive publishing experience: rapid double-blind peer review with constructive feedback.

- Rapid online publication in five weeks: average time from final manuscript arriving in production to online publication.

- Online Open: the option to pay to make your article freely and openly accessible to non-subscribers upon publication on Wiley Online Library, as well as the option to deposit the article in your own or your funding agency's preferred archive (e.g. PubMed). 